\title{
On Minimality and Equivalence of Petri Nets
}

\author{
Annegret K. Wagler, Jan-Thierry Wegener \\ Université Blaise Pascal (Clermont-Ferrand II) \\ Laboratoire d'Informatique, de Modélisation et d'Optimisation des Systèmes \\ BP 10125, 63173 Aubière Cedex, France \\ Annegret.WAGLER@univ-bpclermont.fr wegener@isima.fr
}

\begin{abstract}
The context of this work is the reconstruction of Petri net models for biological systems from experimental data. Such methods aim at generating all network alternatives fitting the given data. To keep the solution set small while guaranteeing its completeness, the idea is to generate only Petri nets being "minimal" in the sense that all other networks fitting the data contain the reconstructed ones.

In this paper, we consider Petri nets with extensions in two directions: priority relations among the transitions of a network in order to allow the modelization of deterministic systems, and control-arcs in order to represent catalytic or inhibitory dependencies. We define a containment relation for Petri nets taking both concepts, priority relations and controlarcs, into account. We discuss the consequences for extended Petri nets differing in their sets of control-arcs and priority relations, and the impact of our results towards the reconstruction of such Petri nets.
\end{abstract}

\section{Introduction}

The aim of systems biology is to analyze and understand different phenomena as, e.g., responses of cells to environmental changes, host-pathogen interactions, or effects of gene defects. To gain the required insight into the underlying biological systems, experiments are performed and the resulting experimental data have to be interpreted in terms of models that reflect the observed phenomena.

Depending on the biological aim and the type and quality of the available data, different types of mathematical models are used and corresponding methods for their reconstruction have been developed, see for instance [9, 12,13 .

Here we focus on Petri nets, a framework which turned out to coherently model both static interactions in terms of networks and the dynamic processes in terms of state changes 1, 2, 10, 15, 17. In fact, a network $(P, T, \mathcal{A}, w)$ reflects the involved components by places $p \in P$ and their interactions by transitions $t \in T$, linked by weighted directed arcs. Each place $p \in P$ can be marked with an integral number of tokens defining a system state $\boldsymbol{x} \in \mathbb{Z}_{+}^{|P|}$, and the studied dynamic processes are represented by sequences of state changes, performed by switching or firing enabled transitions (see Section 1.1).

* The work of the second author was supported by the Labex IMobS3 and the FEDER (Fonds européenne de développement régional). 
To obtain models of this type, we developed in [5,6 64 21] an exact combinatorial approach to reconstruct Petri nets from experimental data in an exclusively data-driven manner.

Our approach takes as input a set $P$ of places and discrete time-series data $\mathcal{X}^{\prime}$ given by sequences $\left(\boldsymbol{x}^{0}, \boldsymbol{x}^{1}, \ldots, \boldsymbol{x}^{m}\right)$ of experimentally observed system states ${ }^{1}$. The goal is to determine all Petri nets $(P, T, \mathcal{A}, w)$ that are able to reproduce the data, i.e., where $T$ contains enough transitions to perform for each $\boldsymbol{x}^{j} \in \mathcal{X}^{\prime}$ the experimentally observed state change to $\boldsymbol{x}^{j+1} \in \mathcal{X}^{\prime}$. This leads to the notion of Petri nets being conformal with the data $\mathcal{X}^{\prime}$; our approach guarantees to generate all of them being minimal in the sense that no superfluous transitions occur in any network, see Section 1.2 .

This combinatorial approach has been extended in two directions 3.14 .

On the one hand, we introduced in $[14,19,20,22$ the concept of priority relations among the transitions of a network in order to allow the modelization of deterministic systems. This means that for states where at least two transitions are enabled, the decision between the different alternatives is not taken randomly, but a specific transition is selected according to additional activation rules ${ }^{2}$. For this purpose, priorities between the transitions of the network can be used (see Section 1.1 for more details). This leads to the notion of $\mathcal{X}^{\prime}$-deterministic Petri nets, which show a prescribed behavior on an experimentally observed subset $\mathcal{X}^{\prime}$ of states: the reconstructed Petri nets $(P, T, \mathcal{A}, w)$ do not only contain enough transitions to reach the experimentally observed successors $\boldsymbol{x}^{j+1}$ from $\boldsymbol{x}^{j}$, but exactly this transition will be selected among all enabled ones in $\boldsymbol{x}^{j}$ which is necessary to reach $\boldsymbol{x}^{j+1}$ (see Section 1.2 .

On the other hand, we adapted the approach from [6] for reconstructing standard Petri nets in another direction, namely to also include control-arcs in order to represent catalytic or inhibitory dependencies [3,4]. Here, an enabled transition $t \in T$ coupled with a read arc (resp. an inhibitory arc) to a place $p \in P$ can switch only if a token (resp. no token) is present in $p$ (see Section 1.1 for more details). This leads to the reconstruction of extended Petri nets which are catalytically conformal with given data.

To keep the solution set small while guaranteeing its completeness, the idea is to generate only Petri nets being "minimal" in the sense that all other networks fitting the data contain the reconstructed ones. For that, it is required to carefully define a notion of minimality taking both concepts, priority relations and controlarcs, into account. The difficulty hereby is that priority relations and control-arcs are concurrent concepts to prevent enabled transitions from switching.

\footnotetext{
${ }^{1}$ Any continuous experimental time-series data need to be discretized in a preprocessing step. As a consequence of this, reconstructing Petri nets, which show exactly the same behavior as a system of ordinary differential equations cannot be expected in general.

${ }^{2}$ In contrast to the normally used stochastic simulation, the aim of this approach is to force the reconstructed Petri net to show the observed behavior of the experiments in a simulation.
} 
The contribution of this paper is to define a containment relation for Petri nets and to discuss the consequences for extended Petri nets differing in their sets of control-arcs and priority relations (Section 2). Finally, we discuss the impact of our results towards the reconstruction of "minimal" $\mathcal{X}^{\prime}$-deterministic extended Petri nets (Section 3).

\section{$1.1 \quad$ Petri Nets}

A Petri net $\mathcal{P}=(P, T, \mathcal{A}, w)$ is a weighted directed bipartite graph with two kinds of nodes, places and transitions. The places $p \in P$ represent the system components (e.g. proteins, enzymes, genes, receptors or their conformational states) and the transitions $t \in T$ stand for their interactions (e.g., chemical reactions, activations or causal dependencies). The $\operatorname{arcs}$ in $\mathcal{A} \subset(P \times T) \cup(T \times P)$ link places and transitions, and the arc weights $w: \mathcal{A} \rightarrow \mathbb{N}$ reflect stoichiometric coefficients of the reactions.

Each place $p \in P$ can be marked with an integral number $x_{p}$ of tokens, and any marking defines a state $\boldsymbol{x} \in \mathbb{N}^{|P|}$ of the system. In biological systems, all components can be considered to be bounded, as the value $x_{p}$ of any state refers to the concentration of the studied component $p \in P$, which can only increase up to a certain maximum $\operatorname{cap}(p)$. This leads to a capacitated Petri net ( $\mathcal{P}$, cap), i.e., a Petri net $\mathcal{P}=(P, T, \mathcal{A}, w)$ together with a capacity function cap $: P \rightarrow \mathbb{N}$, whose set of potential states is $\mathcal{X}:=\left\{\boldsymbol{x} \in \mathbb{N}^{|P|} \mid x_{p} \leq \operatorname{cap}(p)\right\}$.

An extended Petri net $\mathcal{P}=\left(P, T,\left(A_{S} \cup A_{R} \cup A_{I}\right), w\right)$ is a Petri net which has, besides the standard-arcs in $A_{S}$, two additional sets of special arcs (so-called control-arcs): the set of read-arcs ${ }^{3} A_{R} \subset P \times T$ and the set of inhibitor-arcs $A_{I} \subset P \times T$. The set of all control-arcs, i.e., the set $A_{R} \cup A_{I}$, is denoted by $A_{C}$.

A transition $t \in T$ is enabled in a state $\boldsymbol{x} \in \mathcal{X}$ of a capacitated and extended Petri net if all the following conditions are satisfied:

(i) $x_{p} \geq w(p, t)$ for all $p$ with $(p, t) \in A_{S}$,

(ii) $x_{p}+w(t, p) \leq \operatorname{cap}(p)$ for all $p$ with $(t, p) \in A_{S}$,

(iii) $x_{p} \geq w(p, t)$ for all $p$ with $(p, t) \in A_{R}$,

(iv) $x_{p}<w(p, t)$ for all $p$ with $(p, t) \in A_{I}$.

If a transition is not enabled, we say it is disabled. In the case that condition (iii) (resp. (iv) does not hold, we say that $t$ is disabled due to a read-arc (resp. disabled due to an inhibitor-arc).

When a transition $t$ is enabled in a state $\boldsymbol{x}$ it can switch, leading to a successor state $\boldsymbol{x}^{\prime} \in \mathcal{X}$ (denoted by $\boldsymbol{x} \stackrel{t}{\rightarrow} \boldsymbol{x}^{\prime}$ ) whose marking is obtained by

$$
x_{p}^{\prime}:= \begin{cases}x_{p}-w(p, t), & \text { for all } p \text { with }(p, t) \in A_{S}, \\ x_{p}+w(t, p), & \text { for all } p \text { with }(t, p) \in A_{S}, \\ x_{p}, & \text { otherwise. }\end{cases}
$$

\footnotetext{
${ }^{3}$ A read-arc $(p, t) \in A_{R}$, also called test-arc, can be simulated in a standard Petri net by two standard-arcs; namely by the $\operatorname{arcs}(p, t) \in A_{S}$ and $(t, p) \in A_{S}$, where $w(p, t)=w(t, p)$.
} 
In general there can be more than one transition enabled in a state $\boldsymbol{x} \in \mathcal{X}$, and the decision which transition switches is typically taken non-deterministically (and the dynamic behavior is analyzed in terms of reachability, starting from a certain initial state). This is not appropriate for biological system showing a deterministic behavior where, e.g., a certain stimulation always results in the same response. In this case, additional activation rules are required in order to force the switch from a state $\boldsymbol{x}^{j}$ to a specific successor state $\boldsymbol{x}^{j+1}$. For this purpose, priorities between the transitions of the network can be used to determine which of the enabled transitions has to be taken. Note that these priorities typically reflect the rate of the corresponding reactions where the fastest reaction has highest priority. In Marwan et al. [14] it is proposed to model such priorities with the help of partial orders on the set $T$ of transitions of the network $\mathcal{P}$. Here, a partial order $\mathcal{O}$ on $T$ is a relation $\leq$ between pairs of elements of $T$ respecting

- reflexivity (i.e., $t \leq t$ holds for all $t \in T$ ),

- transitivity (i.e., from $t \leq t^{\prime}$ and $t^{\prime} \leq t^{\prime \prime}$ follows $t \leq t^{\prime \prime}$ for all $t, t^{\prime}, t^{\prime \prime} \in T$ ),

- anti-symmetry (i.e., $t \leq t^{\prime}$ and $t^{\prime} \leq t$ implies $t=t^{\prime}$ ).

We call $(\mathcal{P}, \mathcal{O})$ an (extended) Petri net with priorities, if $\mathcal{P}=(P, T, \mathcal{A}, w)$ is an (extended) Petri net and $\mathcal{O}$ a priority relation on $T$.

Note that priorities can prevent enabled transitions from switching: for a state $\boldsymbol{x} \in \mathcal{X}$, let $T(\boldsymbol{x}):=\{t \in T \mid t$ is enabled in $\boldsymbol{x}\}$, then only a transition $t \in T(\boldsymbol{x})$ is allowed to switch or can switch if there is no other transition $t^{\prime} \in T(\boldsymbol{x})$ with $\left(t<t^{\prime}\right) \in \mathcal{O}$. The set of all transitions that are allowed to switch in $\boldsymbol{x}$ is denoted by

$$
T_{\mathcal{P}, \mathcal{O}}(\boldsymbol{x}):=\left\{t \in T(\boldsymbol{x}) \mid \text { there is no } t^{\prime} \in T(\boldsymbol{x}) \text { with }\left(t<t^{\prime}\right) \in \mathcal{O}\right\} .
$$

In this paper we consider capacitated extended Petri nets with priorities $(\mathcal{P}$, cap, $\mathcal{O})$ : extended Petri nets $\mathcal{P}=(P, T, \mathcal{A}, w)$ with a capacity function cap : $P \rightarrow \mathbb{N}$ on their places and a partial order $\mathcal{O} \subset T \times T$ on their transitions.

\subsection{Reconstructing Petri Nets from Experimental Data}

Our main goal is to reconstruct Petri nets from experimental time-series data. We first briefly describe the input, the main idea, and the generated output of the reconstruction approach.

First, a set of components $P$ (later represented by the set of places) is chosen which is expected to be crucial for the studied phenomenon. For each of them, a capacity $\operatorname{cap}(p)$ is given so that all system states are represented by vectors from $\mathcal{X}=\left\{\boldsymbol{x} \in \mathbb{N}^{|P|}, x_{p} \leq \operatorname{cap}(p)\right\}$.

To perform an experiment, the system is stimulated in a state (by external stimuli like the change of nutrient concentrations or the exposition to some pathogens) to generate an initial state $\boldsymbol{x}^{1} \in \mathcal{X}$. Then the system's response to the stimulation is observed and the resulting state changes are measured at certain time points. This yields a sequence $\left(\boldsymbol{x}^{1}, \ldots, \boldsymbol{x}^{k}\right)$ of states $\boldsymbol{x}^{i} \in \mathcal{X}$ reflecting the time-dependent response of the system to the stimulation, denoted by $\mathcal{X}\left(\boldsymbol{x}^{1}\right)=$ 
$\left(\boldsymbol{x}^{1}, \ldots, \boldsymbol{x}^{k}\right)$. Typically, several experiments starting from different initial states in a set Ini $\subseteq \mathcal{X}$ are necessary to describe the whole phenomenon, and we obtain experimental time-series data of the form $\mathcal{X}^{\prime}=\left\{\mathcal{X}\left(\boldsymbol{x}^{1}\right) \mid \boldsymbol{x}^{1} \in\right.$ Ini $\}$, see Example 1 or Example 2 for illustration and $6,7,21$ for more details.

Thus, the experimental setting is given by $\left(P\right.$, cap, $\left.\mathcal{X}^{\prime}\right)$, and the task is to find all networks $\mathcal{P}=\left(P, T, A_{S}, w\right)$ with $P$ as set of places which are appropriate to explain the experimentally observed behavior reported in $\mathcal{X}^{\prime}$.

In the best case, two consecutively measured states $\boldsymbol{x}^{j}, \boldsymbol{x}^{j+1} \in \mathcal{X}^{\prime}$ are also consecutive system states, i.e., $\boldsymbol{x}^{j+1}$ can be obtained from $\boldsymbol{x}^{j}$ by switching a single transition in $T$. This is, however, in general not the case (and depends on the chosen time points to measure the states in $\mathcal{X}^{\prime}$ ), but $\boldsymbol{x}^{j+1}$ is obtained from $\boldsymbol{x}^{j}$ by a switching sequence of some length, where the intermediate states are not reported in $\mathcal{X}^{\prime}$.

In a network $\mathcal{P}$ fitting the experimental data, this can be interpreted as follows. With $\mathcal{P}$, an incidence matrix $M \in \mathbb{Z}^{|P| \times|T|}$ is associated, where each row corresponds to a place $p \in P$ of the network, and each column $M_{. t}$ to the update vector of a transition $t \in T$ :

$$
M_{p t}:= \begin{cases}-w(p, t) & \text { if }(p, t) \in A_{S}, \\ +w(t, p) & \text { if }(t, p) \in A_{S}, \\ 0 & \text { otherwise. }\end{cases}
$$

Reaching $\boldsymbol{x}^{j+1}$ from $\boldsymbol{x}^{j}$ by a switching sequence using the transitions from a subset $T^{\prime} \subseteq T$, is equivalent to obtain the state vector $\boldsymbol{x}^{j+1}$ from $\boldsymbol{x}^{j}$ by adding the corresponding columns $M_{\cdot t}$ of $M$ for all $t \in T^{\prime}$ :

$$
\boldsymbol{x}^{j}+\sum_{t \in T^{\prime}} M_{\cdot t}=\boldsymbol{x}^{j+1} .
$$

Hence, to fit the experimental data, it is required to include enough transitions to perform all experimentally observed switching sequences. More formally, a network $\mathcal{P}=\left(P, T, A_{S}, w\right)$ with incidence matrix $M \in \mathbb{Z}^{|P| \times|T|}$ is conformal with $\mathcal{X}^{\prime}$ if, for each $\boldsymbol{x}^{1} \in$ Ini and any two consecutive states $\boldsymbol{x}^{j}, \boldsymbol{x}^{j+1} \in \mathcal{X}\left(\boldsymbol{x}^{1}\right)$, the linear equation system

$$
\boldsymbol{x}^{j+1}-\boldsymbol{x}^{j}=M \lambda
$$

has an integral solution $\lambda \in \mathbb{N}^{|T|}$ such that $\lambda$ is the incidence vector of a sequence $\left(t^{1}, \ldots, t^{k}\right)$ of transition switches, i.e., there are states $\boldsymbol{x}^{j}=\boldsymbol{y}^{1}, \boldsymbol{y}^{2}, \ldots, \boldsymbol{y}^{k+1}=$ $\boldsymbol{x}^{j+1}$ with $\boldsymbol{y}^{l}+M_{\cdot t^{l}}=\boldsymbol{y}^{l+1}$ for $1 \leq l \leq k$.

This leads to the following problem (Network Reconstruction Problem): Given the experimental setting $\left(P\right.$, cap, $\left.\mathcal{X}^{\prime}\right)$, generate all the networks $\mathcal{P}=\left(P, T, A_{S}, w\right)$ being conformal with $\mathcal{X}^{\prime}$.

For details on the reconstruction approach to solve this problem, we refer to [6. 21]. Note that all returned solution alternatives $\mathcal{P}=\left(P, T, A_{S}, w\right)$ have the same set of places $P$ (as part of the input), but differ in the transition sets $T$ and the corresponding weighted arcs (obtained from all possible combinations of different switching sequences between two consecutively measured 
states $\boldsymbol{x}^{j}, \boldsymbol{x}^{j+1} \in \mathcal{X}^{\prime}$ ). To keep the solution set small while guaranteeing its completeness, the idea is to generate only Petri nets being "minimal" in the sense that removing any transition from $T$ yields a network which cannot explain the experimental observations anymore.

This approach works only to reconstruct standard Petri nets. To refine the dynamic behavior of the reconstructed networks, two different extensions have been considered which both impose further conditions for an enabled transition: priority relations on the transitions to force a deterministic behavior and controlarcs to represent catalytic or inhibitory dependencies.

We next briefly explain the corresponding extensions of the reconstruction approach from [14, 18] and [6. Note that both approaches take the already reconstructed standard networks $\mathcal{P}=\left(P, T, A_{S}, w\right)$ and add either a partial order $\mathcal{O} \subset T \times T$ on their transitions or a set of control-arcs $A_{R} \cup A_{I}$.

To determine a suitable partial order for a network $\mathcal{P}=\left(P, T, A_{S}, w\right)$ being conformal with $\mathcal{X}^{\prime}$,

- consider, for any two consecutively measured states $\boldsymbol{x}^{j}, \boldsymbol{x}^{j+1} \in \mathcal{X}^{\prime}$, the for $\mathcal{P}$ selected sequence of intermediate states $\boldsymbol{x}^{j}=\boldsymbol{y}^{1}, \boldsymbol{y}^{2}, \ldots, \boldsymbol{y}^{k+1}=\boldsymbol{x}^{j+1}$ and

- impose priority relations that force, in all those states $\boldsymbol{y}^{i}$, this transition to have highest priority which is required to reach $\boldsymbol{y}^{i+1}$.

For any such priority relation $\mathcal{O}$, we obtain a (capacitated) Petri net with priorities $(\mathcal{P}$, cap, $\mathcal{O})$ which is $\mathcal{X}^{\prime}$-deterministic, as it shows a prescribed behavior on the experimentally observed subset $\mathcal{X}^{\prime}$ of states.

To reconstruct extended Petri nets, where enzymatic reactions and inhibitions are represented with the help of control-arcs,

- analyze the mass or signal flow experimentally observed in $\mathcal{X}^{\prime}$ in dependency of potential catalytically acting components,

- generate and evaluate a resulting logical formula, and

- insert the corresponding control-arcs in the studied reconstructed network $\mathcal{P}=\left(P, T, A_{S}, w\right)$.

This leads to the reconstruction of (capacitated) extended Petri nets which are catalytically conformal with the given data.

The final goal for the reconstruction is the combination of the two latter methods to a global reconstruction approach producing all $\mathcal{X}^{\prime}$-deterministic extended Petri nets fitting the given data $\mathcal{X}^{\prime}$. To keep the solution set small while guaranteeing its completeness, the idea is again to generate only Petri nets being "minimal" in the sense that all other networks fitting the data contain the reconstructed ones.

While minimality could be easily related to set inclusion of the transition sets of two standard Petri nets, the difficulty for extended Petri nets with priorities is that priority relations and control-arcs are concurrent concepts to prevent enabled transitions from switching. Therefore, it is required to carefully define a notion of minimality taking both concepts, priority relations and control-arcs, into account, which will be the subject of the next section. 


\section{Classification of $\mathcal{X}^{\prime}$-Deterministic Extended Petri Nets}

In this section, we consider capacitated extended Petri nets with priority relations, generated by a reconstruction approach from experimental data $\mathcal{X}^{\prime}$ and, therefore, all being $\mathcal{X}^{\prime}$-deterministic conformal networks. Formalizing a minimality concept for such Petri nets can be done by defining suitable equivalence classes, and selecting a "minimal" one as representative of each equivalence class. Hence, we first have to define an appropriate equivalence relation as well as an inclusion relation for the studied Petri nets, in order to classify the minimal elements w.r.t. inclusion within the equivalence classes.

In the literature there are several concepts for the equivalence of Petri nets, two often used ones are marking equivalence (see, e.g., [8]) and bisimulation equivalence (see, e.g., 11, 16]).

Marking equivalence is defined in terms of reachability in a Petri net $\mathcal{P}=$ $(P, T, \mathcal{A}, w)$. A sequence of transitions $t^{1} \ldots t^{k}$ in $T$ is called a feasible switching sequence for a state $\boldsymbol{x}^{0} \in \mathcal{X}$ in $\mathcal{P}$, if $t^{j} \in T\left(\boldsymbol{x}^{j-1}\right)$ for all $1 \leq j \leq k$. A marking $\boldsymbol{x}^{k}$ is reachable from an initial marking $\boldsymbol{x}^{0}$, if there exists a feasible switching sequence from $\boldsymbol{x}^{0}$ to $\boldsymbol{x}^{k}$. Two Petri nets are marking equivalent, if they have the same set of reachable markings, starting from the same initial marking.

Two Petri nets are bisimilar if every feasible transition sequence in one Petri net is a feasible transition sequence in the other Petri net and vice versa.

The two concepts of marking and bisimulation equivalence are not suitable for our purpose, as we need to compare two $\mathcal{X}^{\prime}$-deterministic conformal networks starting from all initial states $\boldsymbol{x}^{1} \in \operatorname{Ini} \subseteq \mathcal{X}^{\prime}$ (and not just from one initial marking), but only on the states reported in $\mathcal{X}\left(\boldsymbol{x}^{1}\right)=\left(\boldsymbol{x}^{1}, \ldots, \boldsymbol{x}^{k}\right)$ (and not on all intermediate system states between $\boldsymbol{x}^{1}$ and any reachable state $\left.\boldsymbol{x}^{k}\right)$. We, therefore propose to consider for any two consecutively measured states $\boldsymbol{x}^{j}, \boldsymbol{x}^{j+1} \in \mathcal{X}^{\prime}$ only that $\boldsymbol{x}^{j+1}$ is reachable from $\boldsymbol{x}^{j}$ in terms of a feasible transition sequence.

Definition 1. Two $\mathcal{X}^{\prime}$-deterministic extended Petri nets $(\mathcal{P}$, cap, $\mathcal{O}),(\hat{\mathcal{P}}$, cap,$\hat{\mathcal{O}})$ are $\mathcal{X}^{\prime}$-equivalent if both $\mathcal{P}$ and $\hat{\mathcal{P}}$ have the same incidence matrix.

One can easily verify that this is indeed an equivalence relation. Note that all $\mathcal{X}^{\prime}$-deterministic extended Petri nets in the same equivalence class have not only the same set of places (and therefore the same set $\mathcal{X}$ of potential states), but also the sets of transitions, the sets of standard-arcs and their weights are equal. We next define for such Petri nets an inclusion relation, obtained from strengthening the concept of bisimulation equivalence. For that, we call a sequence of transitions $t^{1} \ldots t^{k} \mathcal{O}$-feasible for a state $\boldsymbol{x}^{0}$ of $(\mathcal{P}$, cap, $\mathcal{O})$ if $t^{j} \in T_{\mathcal{P}, \mathcal{O}}\left(\boldsymbol{x}^{j-1}\right)$ for all $1 \leq j \leq k$.

Definition 2. Consider two $\mathcal{X}^{\prime}$-equivalent extended Petri nets $(\mathcal{P}$, cap, $\mathcal{O})$ and $(\hat{\mathcal{P}}$, cap,$\hat{\mathcal{O}})$. We say $\mathcal{P}$ is included in $\hat{\mathcal{P}}$, denoted by $\mathcal{P} \subseteq \hat{\mathcal{P}}$, if and only if for all states $\boldsymbol{x} \in \mathcal{X}$, every $\hat{\mathcal{O}}$-feasible switching sequence for $\boldsymbol{x}$ in $\hat{\mathcal{P}}$ is a $\mathcal{O}$-feasible switching sequence for $\boldsymbol{x}$ in $\mathcal{P}$.

In order to state the definition of "minimal", we need the following notions. For an $\mathcal{X}^{\prime}$-deterministic extended Petri net $\left(\left(P, T, A_{S} \cup A_{C}, w\right)\right.$, cap, $\left.\left.\mathcal{O}\right)\right)$, 
- a control-arc $(p, t) \in A_{C}$ is necessary, if $\left(\left(P, T, A_{S} \cup\left(A_{C} \backslash\{(p, t)\}\right), w\right)\right.$, cap, $\left.\mathcal{O}\right)$ is not conformal to $\mathcal{X}^{\prime}$;

- a priority $\left(t<t^{\prime}\right) \in \mathcal{O}$ is necessary, if $\mathcal{O} \backslash\left\{\left(t<t^{\prime}\right)\right\}$ is not a partial order or $\left(\mathcal{P}\right.$, cap, $\left.\mathcal{O} \backslash\left\{\left(t<t^{\prime}\right)\right\}\right)$ is not $\mathcal{X}^{\prime}$-deterministic;

- a priority $\left(t<t^{\prime}\right) \in \mathcal{O}$ is strictly necessary, if $\left(\mathcal{P}\right.$, cap, $\left.\mathcal{O} \backslash\left\{\left(t<t^{\prime}\right)\right\}\right)$ is not $\mathcal{X}^{\prime}$-deterministic.

If a priority is not (strictly) necessary, we call this element (strictly) unnecessary.

Definition 3. Among all $\mathcal{X}^{\prime}$-equivalent extended Petri nets, $\mathcal{P}$ is minimal iff $\mathcal{P}$ does neither have unnecessary elements nor is included in another Petri net $\hat{\mathcal{P}}$ being $\mathcal{X}^{\prime}$-equivalent to $\mathcal{P}$.

Based on this inclusion relation for $\mathcal{X}^{\prime}$-equivalent Petri nets we compare $\mathcal{X}^{\prime}$ deterministic extended Petri nets. Firstly, we provide a general statement on the inclusion of $\mathcal{X}^{\prime}$-deterministic extended Petri nets.

Lemma 1. Let $(\mathcal{P}, \operatorname{cap}, \mathcal{O})$ and $(\hat{\mathcal{P}}$, cap,$\hat{\mathcal{O}})$ be two $\mathcal{X}^{\prime}$-equivalent Petri nets. Then $\mathcal{P} \subseteq \hat{\mathcal{P}}$ holds iff for all states $\boldsymbol{x} \in \mathcal{X}$ we have $T_{\hat{\mathcal{P}}, \hat{\mathcal{O}}}(\boldsymbol{x}) \subseteq T_{\mathcal{P}, \mathcal{O}}(\boldsymbol{x})$.

In order to classify $\mathcal{X}^{\prime}$-deterministic extended Petri nets for inclusion, we consider $(\mathcal{P}$, cap, $\mathcal{O})$ and $(\hat{\mathcal{P}}$, cap, $\hat{\mathcal{O}})$ with $\mathcal{P}=\left(P, T, A_{S} \cup A_{C}, w\right)$ and $\hat{P}=$ $\left(P, T, A_{S} \cup \hat{A}_{C}, \hat{w}\right)$, respectively, and distinguish the following cases:

- $\mathcal{O} \subset \hat{\mathcal{O}}$ and $A_{C}=\hat{A}_{C}$;

- $\mathcal{O}=\hat{\mathcal{O}}$ and $A_{C} \subset \hat{A}_{C}$

- $\mathcal{O} \subset \hat{\mathcal{O}}$ and $A_{C} \subset \hat{A}_{C}$;

- $\hat{\mathcal{O}} \subset \mathcal{O}$ and $A_{C} \subset \hat{A}_{C}$.

In other words, our aim is to determine, when we can safely remove a priority or a control-arc to get a "smaller" $\mathcal{X}$ '-deterministic extended Petri net.

Lemma 2. Let $(\mathcal{P}, \operatorname{cap}, \mathcal{O})$ and $(\hat{\mathcal{P}}$, cap,$\hat{\mathcal{O}})$ be two $\mathcal{X}^{\prime}$-equivalent Petri nets with $\mathcal{O} \subset \hat{\mathcal{O}}$ and $A_{C}=\hat{A}_{C}$. Then $\mathcal{P} \subseteq \hat{\mathcal{P}}$ holds.

One could be tempted to ask for the converse, i.e., if for two $\mathcal{X}^{\prime}$-equivalent Petri nets $(\mathcal{P}$, cap, $\mathcal{O})$ and $(\hat{\mathcal{P}}$, cap, $\hat{\mathcal{O}})$ with $\mathcal{O}=\hat{\mathcal{O}}$ and $A_{C} \subset \hat{A}_{C}$, the inclusion $\mathcal{P} \subseteq \hat{\mathcal{P}}$ follows. However, it turns out to be not true in general, as the following counter example demonstrates.

Example 1. Consider the two extended Petri nets $(\mathcal{P}, \mathbb{1}, \mathcal{O})$ and $(\hat{\mathcal{P}}, \mathbb{1}, \hat{\mathcal{O}})$ shown on the left and the right side of Figure 1 , respectively. Both Petri nets are $\mathcal{X}_{1}^{\prime}$ equivalent for $\mathcal{X}_{1}^{\prime}$ given by

$$
\begin{aligned}
& \left(\begin{array}{lllll}
1 & 0 & 0 & 0 & 0
\end{array}\right) \rightarrow\left(\begin{array}{lllll}
0 & 0 & 0 & 1 & 0
\end{array}\right), \\
& \left(\begin{array}{lllll}
1 & 1 & 1 & 0 & 0
\end{array}\right) \rightarrow\left(\begin{array}{lllll}
1 & 1 & 0 & 0 & 1
\end{array}\right) \rightarrow\left(\begin{array}{lllll}
0 & 1 & 0 & 1 & 1
\end{array}\right), \\
& \left(\begin{array}{lllll}
0 & 1 & 1 & 0 & 0
\end{array}\right) \rightarrow\left(\begin{array}{lllll}
0 & 1 & 0 & 0 & 1
\end{array}\right)
\end{aligned}
$$

and satisfy $\mathcal{O}=\hat{\mathcal{O}}$ and $A_{C} \subset \hat{A}_{C}$. However, the behavior of both nets differs in a state $\boldsymbol{x}^{1}:=\left(\begin{array}{lllll}1 & 0 & 1 & 0 & 0\end{array}\right)$ (indicated as marking in Figure 1), since we have $T_{\mathcal{P}, \mathcal{O}}\left(\boldsymbol{x}^{1}\right)=\left\{t^{2}\right\}$ and $T_{\hat{P}, \hat{\mathcal{O}}}\left(\boldsymbol{x}^{1}\right)=\left\{t^{1}\right\}$. Thus, neither $\mathcal{P} \subseteq \hat{\mathcal{P}}$ nor $\hat{\mathcal{P}} \subseteq \mathcal{P}$ holds. 

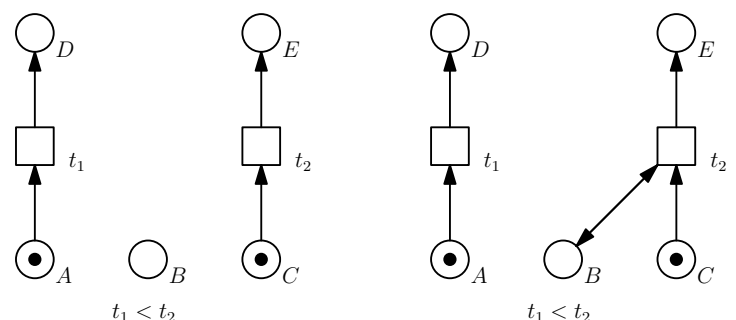

Fig. 1. Two $\mathcal{X}_{1}^{\prime}$-equivalent Petri nets, $(\mathcal{P}, \mathbb{1}, \mathcal{O})$ on the left side and $(\hat{\mathcal{P}}, \mathbb{1}, \hat{\mathcal{O}})$ on the right side, which satisfy $\mathcal{O}=\hat{\mathcal{O}}$ and $A_{C} \subset \hat{A}_{C}$, but are not comparable.

Also in the case, where we have $\mathcal{O} \subset \hat{\mathcal{O}}$ and $A_{C} \subset \hat{A}_{C}$, for two $\mathcal{X}^{\prime}$-equivalent Petri nets $(\mathcal{P}$, cap, $\mathcal{O})$ and $(\hat{\mathcal{P}}$, cap,$\hat{\mathcal{O}})$, neither $\mathcal{P} \subseteq \hat{\mathcal{P}}$ nor $\hat{\mathcal{P}} \subseteq \mathcal{P}$ follows in general. This is demonstrated in the next example.

Example 2. Consider the two extended Petri nets $(\mathcal{P}, \mathbb{1}, \mathcal{O})$ and $(\hat{\mathcal{P}}, \mathbb{1}, \hat{\mathcal{O}})$ shown on the left and the right side of Figure 2 , respectively. Both Petri nets are $\mathcal{X}_{2}^{\prime}$ equivalent for $\mathcal{X}_{2}^{\prime}$ given by

$$
\begin{aligned}
& \left(\begin{array}{llllll}
1 & 1 & 0 & 0 & 0 & 0
\end{array}\right) \rightarrow\left(\begin{array}{llllll}
1 & 0 & 0 & 0 & 1 & 0
\end{array}\right) \rightarrow\left(\begin{array}{llllll}
0 & 0 & 0 & 1 & 1 & 0
\end{array}\right), \\
& \left(\begin{array}{llllll}
1 & 0 & 1 & 0 & 1 & 0
\end{array}\right) \rightarrow\left(\begin{array}{llllll}
1 & 0 & 0 & 0 & 1 & 1
\end{array}\right) \rightarrow\left(\begin{array}{lllll}
0 & 0 & 0 & 1 & 1
\end{array}\right)
\end{aligned}
$$

and satisfy $\mathcal{O} \subset \hat{\mathcal{O}}$ and $A_{C} \subset \hat{A}_{C}$. However, their behavior differs in state $\boldsymbol{x}^{2}:=$

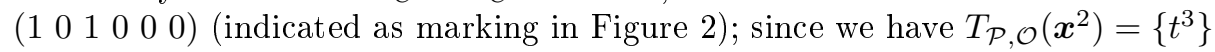
but $T_{\hat{\mathcal{P}}, \hat{\mathcal{O}}}\left(\boldsymbol{x}^{2}\right)=\left\{t^{1}\right\}$. Thus, neither $\mathcal{P} \subseteq \hat{\mathcal{P}}$ nor $\hat{\mathcal{P}} \subseteq \mathcal{P}$ holds.
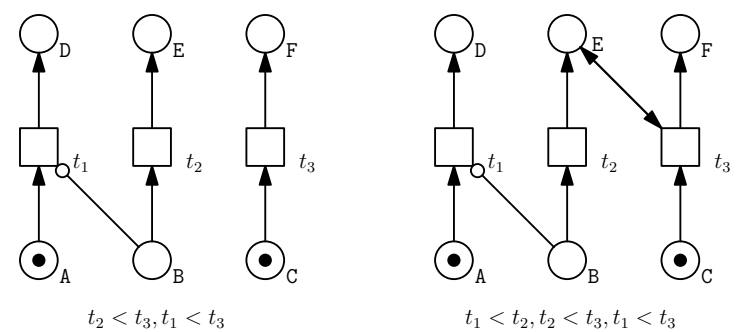

Fig. 2. Two $\mathcal{X}_{2}^{\prime}$-equivalent Petri nets $(\mathcal{P}, \mathbb{1}, \mathcal{O})$ on the left side and $(\hat{\mathcal{P}}, \mathbb{1}, \hat{\mathcal{O}})$ on the right side, which satisfy $\mathcal{O} \subset \hat{\mathcal{O}}$ and $A_{C} \subset \hat{A}_{C}$, but are not comparable.

The next case, which we consider in more detail is $\hat{\mathcal{O}} \subset \mathcal{O}$ and $A_{C} \subset \hat{A}_{C}$. We provide a necessary and a sufficient condition for $\mathcal{P} \subseteq \hat{\mathcal{P}}$ in Theorem 1 and Theorem 2 respectively. 
Theorem 1. Let $(\mathcal{P}$, cap, $\mathcal{O})$ and $(\hat{\mathcal{P}}$, cap,$\hat{\mathcal{O}})$ be two $\mathcal{X}^{\prime}$-equivalent Petri nets with $\hat{\mathcal{O}} \subset \mathcal{O}$ and $A_{C} \subset \hat{A}_{C}$.

If we have $\mathcal{P} \subset \hat{\mathcal{P}}$ then for all $\left(t<t^{\prime}\right) \in \mathcal{O} \backslash \hat{\mathcal{O}}$, where $\left(t<t^{\prime}\right)$ is strictly necessary, $t$ and $t^{\prime}$ are not both enabled in any state in $\hat{\mathcal{P}}$.

Theorem 2. Let $(\mathcal{P}$, cap, $\mathcal{O})$ and $(\hat{\mathcal{P}}$, cap,$\hat{\mathcal{O}})$ be two $\mathcal{X}^{\prime}$-equivalent Petri nets and let $\hat{\mathcal{O}} \subset \mathcal{O}$ and $A_{C} \subset \hat{A}_{C}$. Furthermore, let every control-arc in $\hat{A}_{C}$ be necessary.

If for all $\left(t<t^{\prime}\right) \in \mathcal{O} \backslash \hat{\mathcal{O}}$ the following properties hold:

(i) $t$ and $t^{\prime}$ are not both enabled in any state in $\hat{\mathcal{P}}$,

(ii) there does not exist a transition $t^{\prime \prime}$ with $\left(t^{\prime \prime}<t\right) \in \hat{\mathcal{O}}$,

(iii) $\left(t<t^{\prime}\right)$ is strictly necessary in $\mathcal{P}$,

then $\mathcal{P} \subset \hat{\mathcal{P}}$ follows.

Remark 1. Note that conditions (ii) and (iii) in Theorem 2 are indeed necessary, as there exist counter examples, where the claimed inclusion does not hold anymore, if one of the conditions [ii) or (iii) is dropped.

Remark 2. Finally, note that the presented concepts and approaches work for Petri nets with arbitrary capacities. For the examples, we only consider the binary case to keep the set of potential states small for the required calculations.

\section{Conclusion}

In this work, we address the problem of reconstructing capacitated extended Petri nets with priorities from experimental time-series data $\mathcal{X}^{\prime}$. Already in the case of reconstructing standard Petri nets $[\mathbf{6}$, standard Petri nets with priorities [13] or extended Petri nets without priorities [4], there is typically no unique Petri net being conformal with the given data, but a large set of solution alternatives. We expect that reconstructing $\mathcal{X}^{\prime}$-deterministic extended Petri nets results in even larger solution sets. To keep the solution set small, while still guaranteeing completeness, the idea is to generate only Petri nets being minimal in the sense that all other nets fitting the data contain the reconstructed ones.

While minimality can be easily related to set inclusion of the transition sets of standard Petri nets, the difficulty for extended Petri nets with priorities is that priority relations and control-arcs are concurrent concepts to prevent enabled transitions from switching.

Our contribution is to define a notion of minimality taking both concepts into account. For that, we define when two $\mathcal{X}^{\prime}$-deterministic extended Petri nets are equivalent ( $\mathcal{X}^{\prime}$-equivalence), and provide an inclusion relation for such networks, which allows to determine minimal elements in a class of $\mathcal{X}^{\prime}$-equivalent Petri nets. 
Furthermore, we address the question to classify $\mathcal{X}^{\prime}$-equivalent Petri nets for their inclusion. It turns out that

- the case $\mathcal{O} \subset \hat{\mathcal{O}}$ and $A_{C}=\hat{A}_{C}$ implies $\mathcal{P} \subseteq \hat{\mathcal{P}}$ (Lemma 2),

- there exists a necessary and a sufficient condition (Theorem 1 and Theorem 2. respectively) to conclude from $\hat{\mathcal{O}} \subset \mathcal{O}$ and $A_{C} \subset \hat{A}_{C}$ that $\mathcal{P} \subset \hat{\mathcal{P}}$ holds,

- whereas Petri nets with $\mathcal{O}=\hat{\mathcal{O}}$ and $A_{C} \subset \hat{A}_{C}$ or $\mathcal{O} \subset \hat{\mathcal{O}}$ and $A_{C} \subset \hat{A}_{C}$ are incomparable in general (see Example 1 and Example 2 respectively).

Our further goal is to identify some additional properties for capacitated extended Petri nets with priorities, so that also for the latter two cases some sufficient conditions for their inclusion can be imposed.

Moreover, note that the first case with $\mathcal{O} \subset \hat{\mathcal{O}}$ and $A_{C}=\hat{A}_{C}$ obviously provides a test for inclusion, which can be easily performed.

Also the two conditions from Theorem 1 and Theorem 2 for the case $\hat{\mathcal{O}} \subset \mathcal{O}$ and $A_{C} \subset \hat{A}_{C}$ can be verified efficiently, since the tests whether there is a state, where two transitions $t, t^{\prime}$ are both enabled, as well as that there is no other transition $t^{\prime \prime}$ with $\left(t^{\prime \prime}<t\right)$ can be done in polynomial time by [19.

Hence, here we provided conditions, which imply an inclusion of two $\mathcal{X}^{\prime}$ equivalent Petri Nets, that can indeed be applied practically to reduce the solution set of the studied reconstruction approach.

\section{References}

1. M. Chen and W. Hofestädt. A Petri net application of metabolic processes. J. Syst. Anal. Modell. Simul., 16:113-122, 1994.

2. M. Chen and W. Hofestädt. Quantitative Petri net model fo gene regulated metabolic networks in the cell. In Silico Biology, 3:347-365, 2003.

3. M. Durzinsky, W. Marwan, and A. Wagler. Reconstruction of extended petri nets from time-series data by using logical control functions. Journal of Mathematical Biology, 2012.

4. M. Durzinsky, W. Marwan, and K. A. Wagler. Reconstruction of extended petri nets from time-series data by using logical control functions. Journal of Mathematical Biology, 2011. DOI: 10.1007/s00285-012-0511-3.

5. M. Durzinsky, A. Wagler, and R. Weismantel. A combinatorial approach to reconstruct petri nets from experimental data. In Computational Methods in Systems Biology, volume 5307, pages 328-346, 2008.

6. M. Durzinsky, A. Wagler, and R. Weismantel. An algorithmic framework for network reconstruction. Journal of Theoretical Computer Science, 412(26):2800-2815, 2011.

7. M. Durzinsky, K. A. Wagler, and R. Weismantel. A combinatorial approach to reconstruct petri nets from experimental data. In M. Heiner and A.M. Uhrmacher, editors, CSMB, volume 5307 of Lecture Notes in Computer Science, pages 328-346, 2008.

8. J. Esparza and M. Nielsen. Decidability Issues for Petri Nets - a Survey. Bulletin of the European Association for Theoretical Computer Science, 52:245-262, 1994. 
9. Boris N. Kholodenko, Anatoly Kiyatkin, Frank J. Bruggeman, Eduardo Sontag, Hans V. Westerhoff, and Jan B. Hoek. Untangling the wires: A strategy to trace functional interactions in signaling and gene networks. Proceedings of the National Academy of Sciences, 99(20):12841-12846, October 2002.

10. I. Koch and M. Heiner. Petri nets. In B. H. Junker and F. Schreiber, editors, Biological Network Analysis, Wiley Book Series on Bioinformatics, pages 139-179, 2007.

11. M. Kot and Z. Sawa. Bisimulation equivalence of a bpp and a finite-state system can be decided in polynomial time. Electron. Notes Theor. Comput. Sci., 138(3):49-60, December 2005.

12. R. Krishna and S. Guo. A partial granger causality approach to explode causal networks derived from multi-parameter data. Computational Methods in Systems Biology, 5307:9-27, 2008.

13. R. Laubenbacher and B. Stigler. A computational algebra approach to reverse engineering of gene regulatory networks. Journal of Theoretical Biology, 229:523$537,2005$.

14. W. Marwan, A. Wagler, and R. Weismantel. A mathematical approach to solve the network reconstruction problem. Math. Methods of Operations Research, 67(1):117-132, 2008.

15. W. Marwan, A. Wagler, and R. Weismantel. Petri nets as a framework for the reconstruction and analysis of signal transduction pathways and regulatory networks. Natural Computing, 10:639-654, 2011.

16. D. Park. Concurrency and automata on infinite sequences. In Proceedings of the 5th GI-Conference on Theoretical Computer Science, pages 167-183, London, UK, UK, 1981. Springer-Verlag.

17. J. W. Pinney, R. D. Westhead, and G. A. McConkey. Petri net representations in systems biology. Biochem. Soc. Tarns., 31:1513-1515, 2003.

18. L. M. Torres and K. A. Wagler. Model reconstruction for discrete deterministic systems. Electronic Notes in Discrete Mathematics, 36:175-182, 2010.

19. L. M. Torres and K. A. Wagler. Encoding the dynamics of deterministic systems. Math. Methods of Operations Research, 73:281-300, 2011.

20. L. M. Torres, K. A. Wagler, and R. Weismantel. Modelling the dynamic behavior of deterministic biological systems (extended abstract). Proc. of ALIO/EURO Workshop on Appl. Comb. Opt., 2008. ISBN 978-950-29-1116-8.

21. K. A. Wagler. Prediction of network structure, volume 16 of Computational Biology, pages 309-338. Springer London, 2010.

22. K. A. Wagler and R. Weismantel. The combinatorics of modeling and analyzing biological systems. Natural Computing, 10:655-681, 2011. 